\title{
A brief history of short wavelength coherent radiation sources
}

Greg Tallents

Greg Tallents, "A brief history of short wavelength coherent radiation sources," Proc. SPIE 11886, International Conference on X-Ray Lasers 2020, 1188605 (8 July 2021); doi: 10.1117/12.2585789

SPIE. Event: XVII International Conference on X-Ray Lasers, 2020, Online Only 


\title{
A brief history of short wavelength coherent radiation sources
}

\author{
Greg. Tallents \\ York Plasma Institute, Department of Physics, University of York, York YO10 5DD, U.K.
}

\begin{abstract}
Almost contemporaneously with the building of the first optical laser at wavelength $694 \mathrm{~nm}$ in 1960, theoretical speculation and experiments were underway to produce lasing at shorter wavelengths in the x-ray range. This paper presents a brief review of plasma-based x-ray lasers. Plasma-based x-ray laser research has increased understanding of the atomic physics of plasmas and developed a suite of short wavelength lasers suitable for applications. The history of the development of soft x-ray lasers created by the irradiation of solid targets with pumping optical pulses is examined after setting the work in context with a summary of work covering a broad range of short wavelength coherent sources. The results of methods developed to characterise plasmas-based soft x-ray lasers are discussed.
\end{abstract}

Keywords: X-ray laser, plasma laser, EUV, XUV, recombination laser, collisionally pumped laser

\section{INTRODUCTION}

The building of the first optical laser ${ }^{1}$ at wavelength $694 \mathrm{~nm}$ in 1960 almost immediately generated theoretical speculation and experiments aimed at producing lasing at shorter wavelengths in the x-ray range. This paper aims to review the work on recombination and collisionally pumped soft-X-ray lasers where a laser-produced plasma formed from a solid target is used as the lasing medium. Research developments over 40 years have moved such plasma-based soft-X-ray lasers from large laboratories with inertial fusion scale pumping lasers, to table-top laser systems capable of rapid pulse rates and the production of small-laboratory/commercial scale high average power soft X-ray laser output.

Soft x-ray lasers using a solid-target laser-produced plasma as the lasing medium are created by focusing an optical laser to a line of dimensions typically $2-50 \mathrm{~mm}$ in length and $<100 \mu \mathrm{m}$ width on the target so that lasing occurs along the line in the plasma expanding normally to the target surface. An introduction to a broader range of short wavelength coherent sources is first presented, the development of soft x-ray lasers using solid target laser-produced plasmas as the lasing medium is then summarised and finally the results of methods developed to characterise plasma-based soft x-ray lasers are discussed.

\section{A SUMMARY OF SHORT WAVELENGTH COHERENT LIGHT GENERATION}

In this Section, a range of methods developed to produce coherent light at wavelengths shorter than the ultraviolet are briefly discussed. At wavelengths longer than the hard x-ray (say wavelengths $>0.1 \mathrm{~nm}$ ) and shorter than the ultraviolet (say wavelengths $<50 \mathrm{~nm}$ ), the spectral range is known as the extreme ultra-violet (with acronyms of XUV or EUV) or soft x-ray. Books dealing with this intermediate spectral range were published in 1990 and 2006 and are useful references to the development of short wavelength coherent sources. ${ }^{2,3}$ A comprehensive textbook on the technologies and applications of the extreme ultraviolet is available. ${ }^{4}$

Focusing optical lasers into non-linear media produces useful coherent harmonic output at short wavelengths, but efficiencies are typically small $\left(\approx 10^{-6}\right)$ and the harmonic process typically requires $<100$ fs laser pulses so the time-averaged short wavelength output power is limited. ${ }^{5,6}$ Coherent soft x-ray output can be produced by wakefield acceleration, where a laser pulse propagates through a near critical-density plasma formed in a gas jet. Electrons are accelerated to relativistic velocities in a bubble formed behind the propagating pulse and oscillate producing harmonic 'betatron' output in the soft x-ray in a narrow beam of divergence $\propto 1 / \gamma$, where $\gamma$ is the

Further author information: (Send correspondence to)

E-mail: greg.tallents@york.ac.uk

International Conference on X-Ray Lasers 2020, edited by Davide Bleiner,

Proc. of SPIE Vol. 11886, $1188605 \cdot$ ( ) 2021 SPIE · CCC code:

0277-786X/21/\$21 - doi: $10.1117 / 12.2585789$

Proc. of SPIE Vol. 11886 1188605-1 
Lorentz factor for the electrons. The source size for betatron radiation is $\approx$ micron size and the driving laser pulses are of femtosecond duration, so the source is bright, but with a small/modest energy per pulse. ${ }^{7,8}$

Due to the high energy density required to pump a transition with high photon energy, studies aimed at achieving high energy per pulse output at short wavelengths have involved the production of high temperature plasma. A high energy per pulse enables high average power output when the source is driven at a high rep rate. Successful mechanisms to produce short wavelength lasing at first centered on a recombination approach, where a hot plasma formed in a picosecond duration optical laser-plasma is rapidly cooled leading to preferential population of higher level quantum states and population inversion. ${ }^{9,10,11,12,13}$ Pumping geometries employed for recombination laser research were varied with, for example, pumping optical pulses incident into plasma or gas producing the initial free electrons by optical field ionization ${ }^{12}$ or optical lasers focussed to a line on a solid target. $^{13}$

In the 1980s, a new approach involving collisional excitation of closed-shell ionic configurations (neon-like and nickel-like ions) achieved high output saturated lasing where stimulated emission significantly depletes the upper quantum state. ${ }^{14,15,16}$ In the 1990s to 2000s, much experimental effort extended the range of plasmas where these collisionally-pumped lasers operated. ${ }^{17,18,19}$ A pre-pulse and grazing-incidence input of the main pumping pulse was found to increase the absorption and to deposit the pumping laser energy at the optimum density. This allowed lasing at short wavelengths with pumping from high rep rate optical lasers of duration several picoseconds with energy less than one Joule per pulse: conditions enabling table-top short wavelength laser action. ${ }^{20,21,22}$

Capillary lasers were also developed where a sequence of electrical discharges through argon contained in a narrow ceramic capillary produces the necessary plasma energy density and electron collisional pumping to produce lasing at $46.9 \mathrm{~nm}$ in neon-like argon. ${ }^{23,24}$ Optical field ionization using circularly polarized laser light incident into a gas or pre-formed plasma has also produced electron collisionally pumped lasing at similar wavelengths, for example, $32.8 \mathrm{~nm}$ in nickel-like krypton. ${ }^{25,26}$

X-ray free electron lasers first demonstrated ${ }^{27}$ in 2010 exploit the high energy density of electrons accelerated to relativistic energies in the type of large-scale devices previously used for particle physics experiments. A ponderomotive force (due to the high laser radiation energy density) causes electrons to microbunch so that an undulator (a periodic spatially oscillating magnetic field) accelerates electrons to produce coherent high power x-ray output proportional to the square of the number of magnetic oscillations and the square of the number of electrons in the microbunch. X-ray output with photon energies $\approx 10 \mathrm{keV}$ in pulses of $1-100 \mathrm{fs}$ duration are now routinely used for applications at a number of $\mathrm{x}$-ray free electron laser facilities.

\section{PLASMA-BASED LASERS}

Rapid recombination in a cooling plasma can produce population inversions. Electrons preferentially recombine into high-lying quantum state which are closest to their initial energy in the continuum of free electrons. Studies of excited state populations in ions ${ }^{28}$ show that there is a 'bottle-neck' excited quantum state where for higher energy states the de-populating processes are collisional, while lower-lying quantum states have populations where the de-populating process is radiative decay to lower states. If collisions dominate the de-population of a quantum state, the populations will have Boltzmann population ratios and so a population inversion cannot occur between states with energies above the bottle-neck quantum state. The ideal density for a plasma laser is where the bottle-neck state of the lasing ion lies below the upper lasing quantum state and above the lower lasing quantum state. There is thus an optimum density for short wavelength lasing in plasma ionic material at electron densities where the bottle-neck state satisfies this criteria.

Evidence for gain from plasma material with recombination as the pumping mechanism has been observed, ${ }^{11,29}$ but saturated output has not been obtained with recombination as the sole laser pumping process. Saturated lasing occurs when stimulated emission depletes the upper quantum state population and reduces the gain coefficient. Interestingly, there is modeling evidence ${ }^{15}$ that the gains in the first observations of nominally collisionally-pumped $\mathrm{x}$-ray lasers between $3 \mathrm{p}-3 \mathrm{~s}$ states in neon-like selenium were augmented by recombination. These first collisionally pumped $\mathrm{x}$-ray lasers ${ }^{14,15}$ required thin targets irradiated at intensities $\approx 10^{14} \mathrm{Wcm}^{-2}$ by the Novette laser so that the plasmas cooled rapidly after irradiation. Contrary to expectations, the $J=2 \rightarrow 1$ 


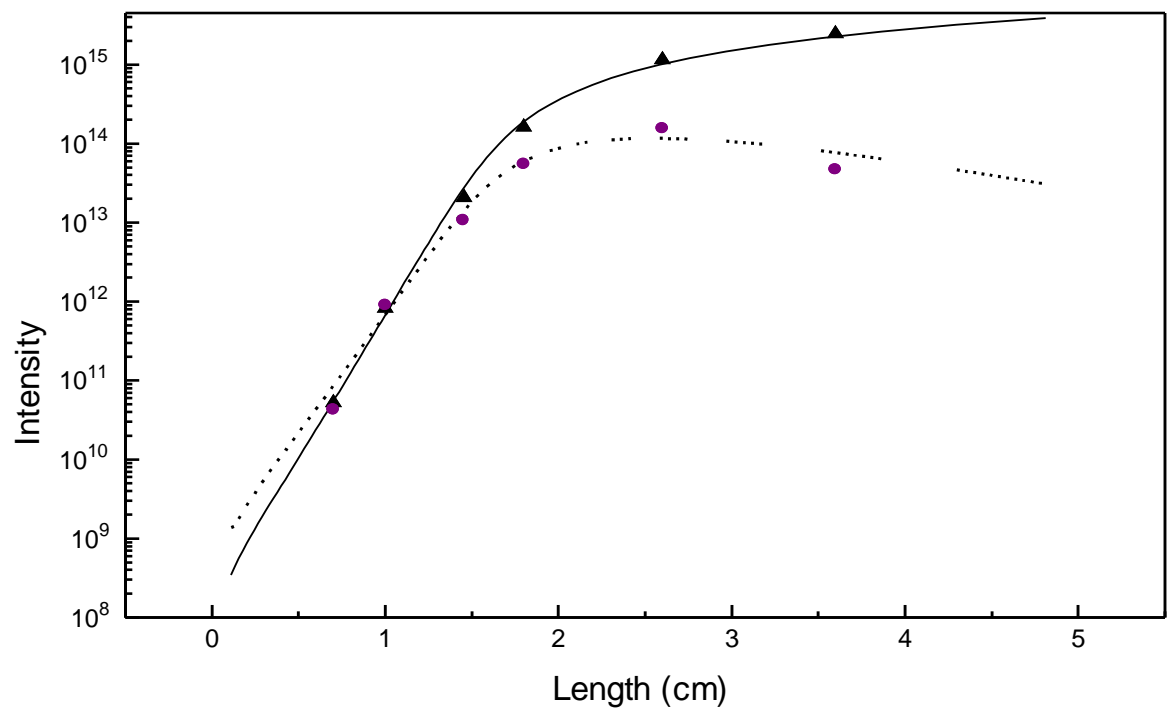

Figure 1. The output at $5.86 \mathrm{~nm}$ (solid line and triangles) and $6.37 \mathrm{~nm}$ (broken line and circles) of a plasma-based soft X-ray laser as a function of plasma length. The intensity scale is in $\mathrm{Wcm}^{-2}$ at the plasma deduced using a model (continuous curves) fitted to the data points. The model small signal gain coefficients deduced from the exponential increase in intensity at shorter lengths are $9 \mathrm{~cm}^{-1}(5.86 \mathrm{~nm})$ and $7.5 \mathrm{~cm}^{-1}(6.37 \mathrm{~nm})$ with intensity saturation occuring at plasma length $\approx 2 \mathrm{~cm}$.

transitions at $20.6 \mathrm{~nm}$ and $20.9 \mathrm{~nm}$ were found to lase rather than the $J=0 \rightarrow 1$ transition at $18.3 \mathrm{~nm}$. This is consistent with significant recombination as recombination cascades from higher states (for example, $3 \mathrm{~d}$ with $J=$ 3 ) will preferentially populate the upper $J=2$ states and not the $J=0$ state (for example, $J=3 \rightarrow 0$ radiative decays are forbidden).

Initial successes with collisionally pumped $\mathrm{x}$-ray lasers ${ }^{14,15}$ required the large Novette laser, but led to developments where much smaller laser systems successfully produced plasma-based soft x-ray lasing. It was found that using a pre-pulse to first irradiate the solid targets increased soft x-ray laser output. ${ }^{30}$ As with the recombination lasers, gain with collisionally-pumped lasers cannot be produced if the bottleneck state is below the lower lasing level. The optimum density for gain is therefore over a small range of electron densities close to the condition where the bottleneck state lies above the lower lasing level. Using a pre-pulse means that the density gradients from the expanding plasma are smaller when the main pumping pulse is incident and so a larger volume of plasma is in the optimum density range for gain. The x-ray laser output is also less refracted by the smaller density gradient and more likely to remain close to the densities of optimum gain. With a pre-pulse, thick slab targets were found to be suitable and the use of thin targets ${ }^{14}$ was no longer necessary.

Moving to low density gradient plasmas created using a pre-pulse ensured that the rate of recombination is reduced. With reduced recombination, the $J=0 \rightarrow 1$ transitions in neon-like ions became the predominant lasing output. ${ }^{31}$ In addition, atomic physics calculations showed that lasing in nickel-line ions (rather than neonlike) has higher gain. Using pre-pulses, x-ray laser gain on 4d- 4p $J=0 \rightarrow 1$ transitions in several nickel-like ions were observed. ${ }^{18}$

Improving the efficiency of pumping with pre-pulses enabled the shortest wavelength plasma-based saturated laser to be achieved in nickel-like dysprosium at $5.86 \mathrm{~nm}$ using the Vulcan laser. ${ }^{19}$ A peculiarity of the atomic 


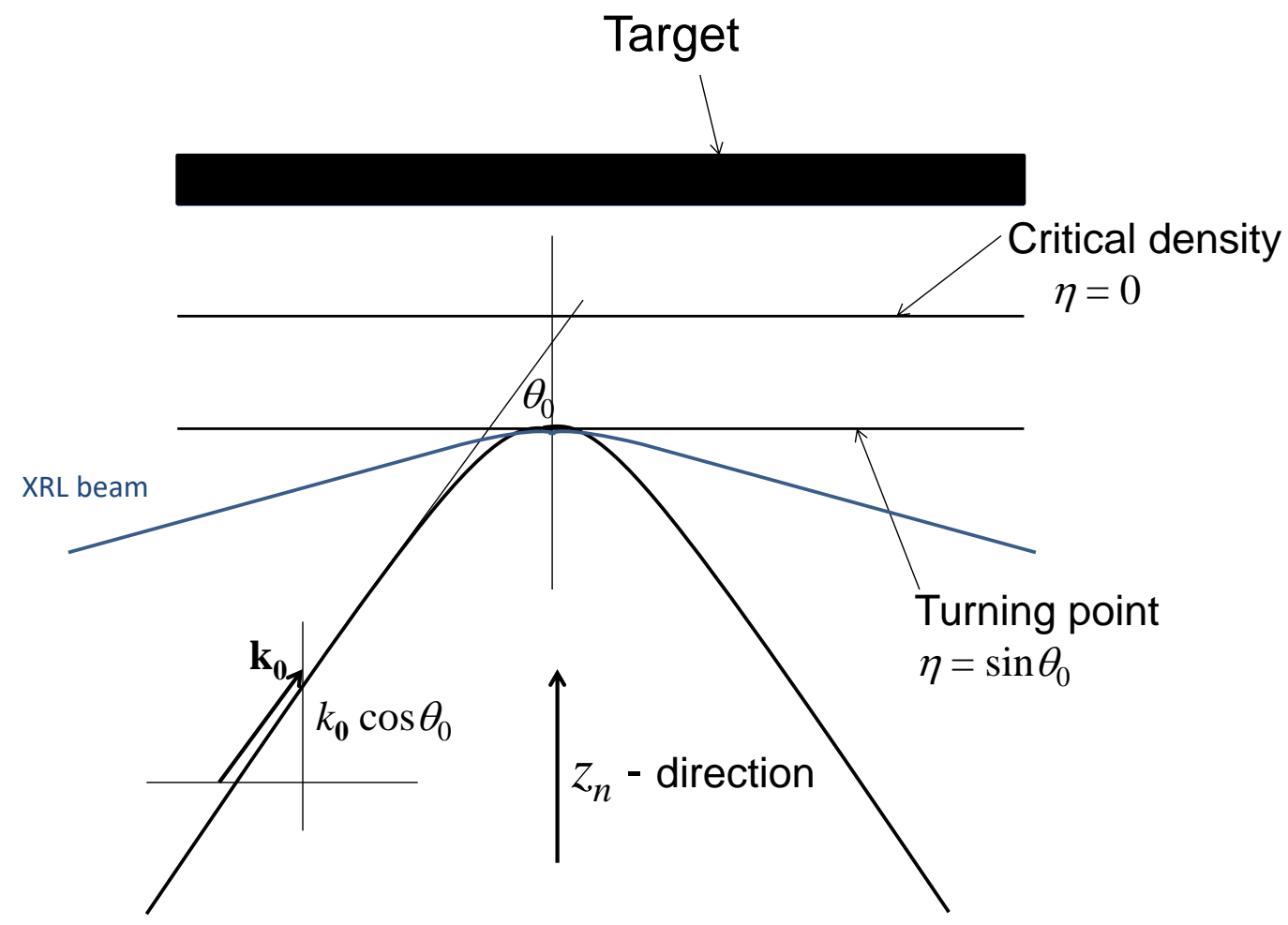

Figure 2. A schematic of pumping rays (black) incident into an expanding plasma produced from a solid target and the ray path taken by a soft x-ray beam. Light penetrates to a turning point with refractive index $\eta=\sin \theta_{0}$, where $\theta_{0}$ is the angle of incidence. It is possible to have the same turning point for the soft x-ray beam and the optical beam which enables optimization of the soft x-ray laser gain. (Figure adapted from Tallents ${ }^{28}$ ).

physics of nickel-like ions causes Ni-like Dy to have approximately equal values of gain coefficient for two $4 \mathrm{~d}-$ $4 \mathrm{p} J=0 \rightarrow 1$ transitions associated with different lower laser level quantum states (an energy state $\left(3 \mathrm{~d}_{5 / 2}\right.$, $\left.4 \mathrm{p}_{3 / 2}\right) J=1$ and an energy state $\left.\left(3 \mathrm{~d}_{3 / 2}, 4 \mathrm{p}_{1 / 2}\right) J=1\right)$. The upper laser state is $\left(3 \mathrm{~d}_{3 / 2}, 4 \mathrm{~d}_{3 / 2}\right) J=0$ for both transitions. As the upper laser level is the same for both lasing transitions, saturation of the slightly higher gain line at $5.86 \mathrm{~nm}$ causes the gain coefficient for the other lasing line at $6.37 \mathrm{~nm}$ to also reduce (see Figure 1). Lower atomic number $\mathrm{Ni}$-like ions have gain predominantly on the lower wavelength transition (lower lasing level $\left.\left(3 \mathrm{~d}_{5 / 2}, 4 \mathrm{p}_{3 / 2}\right) J=1\right)$ so that in saturation, the laser output is essentially monochromatic.

A further and significant improvement to the efficiency of plasma-based soft x-ray lasers was introduced by irradiating pre-pulse plasmas with the main pumping pulse at a grazing angle of incidence. Firstly, grazingincidence irradiation ensures that the laser heats the optimum density of plasma for gain. Incoming pump laser pulses penetrate to a turning point as illustrated in Figure 2. Higher density plasma closer to the critical density is not needlessly heated. The enhanced soft-x-ray laser output can amplify with minimal refraction effect in densities close to the optimum density. There is also another advantage with grazing-incidence pumping. With typical gain duration of $\Delta t$ of $\approx 1-10$ picoseconds, amplification of a soft x-ray laser pulse only occurs over lengths $c \Delta t \approx 0.3-3 \mathrm{~mm}$ and lengths of $10-20 \mathrm{~mm}$ are needed for saturation. Traveling wave pumping where the pumping optical laser pulse moves along the target at close to the speed of light $c$ was found to increase the efficiency of laser output. ${ }^{32}$ Grazing-incidence irradiation introduces an intrinsic traveling wave pumping along the target length. The development of grazing-incidence pumping has enabled soft-x-ray lasing down to Ni-like Dy at $5.86 \mathrm{~nm}$ to be observed with pumping from optical laser pulses of energy $\approx 1-10$ Joule. ${ }^{21,22}$ 


\section{PROPERTIES OF PLASMA-BASED LASERS}

Soft x-ray lasers pumped by optical pulses with a laser-produced plasmas as the amplifying medium have typical pulse durations $\Delta t$ of $\approx 1-10$ picoseconds largely independent of the duration of the pumping laser pulse duration. Optical lasers of several picosecond duration are consequently optimum for pumping. Due to the short duration of the gain, it is not possible to create a laser cavity as amplification only occurs over lengths $c \Delta t \approx 0.3-3 \mathrm{~mm}$. For significant gain-length product, plasma lengths needed for saturation are typically in the range $10-20 \mathrm{~mm}$ (see for example Figure 1) and cavity mirrors cannot be readily set apart by the necessary $0.3-3 \mathrm{~mm}$ as such proximity to the plasma would result in mirror damage on each shot. In addition, though well designed multilayer mirrors can reflect up to $70 \%$ at normal incidence in the soft x-ray regime, the construction of output mirrors with partial transmission to form a laser cavity is difficult. The closest approach to forming a cavity for soft x-ray lasing has involved a single mirror enabling a double pass through the plasma amplifying medium. ${ }^{33}$ Plasma-based soft x-ray lasers usually operate as amplified spontaneous emission (ASE) lasers where some spontaneous emission propagating along the gain medium is amplified by stimulated emission. A simple theoretical explanation of this ASE operation has been developed. ${ }^{34,35}$

A several picosecond duration of gain ensures that the soft x-ray laser pulse durations are of picosecond duration as there is some laser output pulse shortening compared to the gain duration as the laser intensity amplification exponentiates the gain coefficient profile. ${ }^{36}$ Measurements have shown that the longitudinal coherence length of soft x-ray lasers approaches the pulse duration and so has picosecond temporal coherence. ${ }^{37,38}$ As the longitudinal coherence length is of picosecond duration, the transform limit of the spectral bandwidth $\Delta \nu$ of soft x-ray laser output is very narrow. We can estimate assuming the transform limit that $\Delta \nu / \nu \approx 10^{-4}-10^{-5}$. Calculations and measurements of line broadening of the lasing lines are in agreement with such spectral bandwidths. ${ }^{39}$ A spectral bandwidth $\Delta \nu / \nu \approx 10^{-4}-10^{-5}$ gives an extremely narrow spectral range that could be used for applications requiring an extremely well-defined and spectrally narrow laser wavelength, but to the author's knowledge such applications have not been explored.

The transverse coherence of plasma-based soft x-ray lasers has been measured using, for example, Young's slit arrangements. ${ }^{40,41}$ The soft x-ray laser beams are often dominated by speckle associated with individual spontaneous emission events that are amplified. ${ }^{42}$ Effective methods to improve the transverse coherence have involved the seeding of the amplification from harmonic radiation generated in a gas jet, ${ }^{38,43}$ using two plasma amplifiers in an 'ASE oscillator'/amplifier arrangement ${ }^{44,45}$ and employing a mirror to double pass a plasma amplifier. $^{33}$

\section{CONCLUSIONS}

The development of plasma-based soft X-ray lasers over approximately 40 years has been summarised with an emphasis on the techniques which have enabled table-top soft X-ray laser pumping with $\approx$ Joule energy pulses. Lasing down to wavelength $5.86 \mathrm{~nm}$ has been observed with table-top laser systems, enabling high average power operation. The use of laser pre-pulses and grazing-incidence pumping has ensured that optimization of gain coefficients and gain amplification with minimal refraction loss can occur. Pre-pulses reduce plasma gradients which increase the volume of the gain region and reduce refraction of the x-ray laser beam out of the gain region. Grazing-incidence pumping enables the pump laser pulse to heat the optimum gain region without wasting energy heating plasma closer to the critical density where gain cannot occur. Some of the key properties of plasma-based soft x-ray lasers have been summarised.

\section{ACKNOWLEDGMENTS}

The author acknowledges many students, researchers, and colleagues involved in the development of short wavelength lasers with which he has collaborated: including Frank Irons, Geoff Pert, Mike Key, Ciaran Lewis, Steve Rose, Jie Zhang, Justin Wark, Carmen Menoni and Jorge Rocca. I apologize to many authors who worked on plasma-based soft-X-ray lasers and whose contributions have not been cited due to the short nature of this review. Readers can find many of these contributions in the references of the listed citations. 


\section{REFERENCES}

[1] T. H. Maiman, "Stimulated optical radiation in ruby," Nature 187, pp. 493-494, 1960.

[2] R. Elton, X-ray lasers, Academic Press, Amsterdam, 1990.

[3] P. Jaegle, Coherent sources of XUV radiation: soft $x$-ray lasers and high-order harmonic generation, Springer-Verlag, New York, U.S.A., 2006.

[4] D. Attwood, Soft x-rays and extreme ultraviolet radiation, Cambridge University Press, Cambridge, U.K., 1999.

[5] P. B. Corkum, "Plasma perspectives on strong field multiphoton ionization," Phys. Rev. Lett. 71, pp. 19941997, 1993.

[6] P. Gibbon, "High harmonic generation in plasmas," IEEE J. Quant. Elect. 33, pp. 1915-1924, 1997.

[7] Y. Glinec, J. Faure, and A. Lifschitz et al, "Direct observation of betatron oscillations in a laser-plasma electron accelerator," Eur. J. Phys. 81, p. 64001, 2008.

[8] M. Schnell, A. Saert, and B. Landgraf et al, "Deducing the electron-beam diameter in a laser-plasma accelerator using x-ray betatron radiation," Phys. Rev. Lett 108, p. 075001, 2012.

[9] G. J. Tallents, "Population inversion for soft x-ray transitions computed for rapidly cooling plasmas," $J$. Phys. B 10, pp. 1769-1780, 1977.

[10] G. J. Tallents, "Effect of Lyman-alpha self absorption on population inversions between quantum states 2 and 3 of hydrogen-like ions in recombing plasmas," J. Phys. B 11, pp. L157-L161, 1978.

[11] S. Suckewer, C. H. Skinner, and H. Milchberg et al, "Amplification of stimulated soft-x-ray emission in a confined plasma-column," Phys. Rev. Lett 55, pp. 1753-1756, 1985.

[12] Y. Nagata, K. Midorikawa, and S. Kubodera et al, "Soft x-ray amplification of the Lyman-alpha transition by optical-field-induced ionization," Phys. Rev. Lett. 71, pp. 3774-3777, 1993.

[13] J. Zhang, M. H. Key, and P. A. Norreys et al, "Demonstration of high-gain in a recombination XUV laser at $18.2 \mathrm{~nm}$ driven by a 20-J, 2 ps glass-laser," Phys. Rev. Lett. 74, pp. 1335-1338, 1995.

[14] D. L. Matthews, P. L. Hagelstein, and M. D. Rosen et al, "Demonstration of a soft x-ray amplifier," Phys. Rev. Lett. 54, pp. 110-113, 1985.

[15] M. D. Rosen, P. L. Hagelstein, and E. M. Campbell et al, "Exploding-foil technique for achieving a soft x-ray laser," Phys. Rev. Lett. 54, pp. 106-109, 1985.

[16] G. J. Tallents, "The physics of soft x-ray lasers pumped by electron collisions in laser plasmas," J. Phys. D 36, pp. R259-R276, 2003.

[17] A. Carillon, H. Z. Chen, and P. Dhez et al, "Saturated and near-diffraction-limited operation of an xuv laser at $23.6 \mathrm{~nm}, "$ Phys. Rev. Lett. 68, pp. 2817-2920, 1992.

[18] J. Zhang, A. G. MacPhee, and J. Lin et al, "A saturated x-ray laser beam at 7 nanometers," Science 276, pp. 1097-1100, 1997.

[19] R. Smith, G. J. Tallents, and J. Zhang et al, "Saturation behavior of two x-ray lasing transitions in Ni-like Dy," Phys. Rev. A 59, pp. R47-R50, 1999.

[20] R. Keenan, J. Dunn, and P. K. Patel et al, "High repetition rate grazing incidence pumped x-ray laser operating at 18.9 nm," Phys. Rev. Lett. 94, p. 103901, 2005.

[21] B. A. Reagan, M. Berrill, and K. A. Wernsing et al, "High-average-power, 100 hz repetition rate, tabletop soft-x-ray lasers at sub-5-nm wavelengths," Phys. Rev. A 89, p. 053820, 2014.

[22] A. Rockwood, Y. Wang, and S. Wang et al, "Compact gain-saturated x-ray lasers down to $6.85 \mathrm{~nm}$ and amplification down to $5.85 \mathrm{~nm}, "$ Optica 5, pp. 257-262, 2018.

[23] J. J. Rocca, Shlyaptsev, and F. G. Tomasel et al, "Demonstration of a discharge pumped table-top soft x-ray laser," Phys. Rev. Lett. 73, pp. 2192-2195, 1994.

[24] B. R. Benware, C. D. Macchietto, and C. H. Moreno et al, "Demonstration of a high average power table-top soft x-ray laser," Phys. Rev. Lett. 81, pp. 5804-5807, 1998.

[25] S. Sebban, T. Mocek, and D. Ros et al, "Demonstration of a Ni-like Kr optical-field-ionization collisional soft x-ray laser at 32.8 nm," Phys. Rev. Lett. 89, p. 253901, 2002.

[26] P. Zeitoun, G. Faivre, and S. Sebban et al, "A high-intensity highly coherent soft x-ray femtosecond laser seeded by a high harmonic beam," Nature 431, pp. 426-429, 2004. 
[27] P. Emma, R. Akre, and J. Arthur et al, "First lasing and operation of an angstrom-wavelength free-electron laser," Nature Photonics 4, pp. 641-647, 2010.

[28] G. J. Tallents, An introduction to the atomic and radiation physics of plasmas, Cambridge University Press, Cambridge, U.K., 2018.

[29] D. V. Korobkin, C. H. Nam, and S. Suckewer et al, "Demonstration of soft x-ray lasing to ground state in LiIII," Phys. Rev. Lett 77, pp. 5206-5209, 1996.

[30] A. Behjat, J. Lin, and G. J. Tallents et al, "The effects of multi-pulse irradiation on x-ray laser media," Optics Commun. 135, pp. 49-54, 1997.

[31] J. Zhang, S. T. Chunyu, and Y. L. Lou et al, "Soft-x-ray lasing at $32.6 \mathrm{~nm}$ in Ne-like Ti ions driven by 40 J of energy from two 650-ps laser pulses," Phys. Rev. A 53, pp. 3640-3646, 1996.

[32] J. Lin, G. Tallents, and A. MacPhee et al, "Travelling wave chirped pulse amplified transient pumping for collisional excitation lasers," Optics Commun. 166, pp. 211-218, 1999.

[33] B. Rus, T. Mocek, and A. R. Prag et al, "Multimillijoule highly coherent x-ray laser at $21 \mathrm{~nm}$ operating in deep saturation through double-pass amplification," Phys. Rev. A 66, p. 063806, 2002.

[34] G. J. Pert, "Output characteristics of amplified-stimulated-emission lasers," J. Opt. Soc. Am. B 11, pp. 1425-1435, 1994.

[35] G. J. Tallents, G. Eker, and F. Strati et al, "Saturated x-ray lasers," in Soft X-ray lasers and applications III, J. J. Rocca and L. B. DaSilva, eds., Proc. SPIE 3776, pp. 75-82, 1999.

[36] Y. Abou-Ali, G. J. Tallents, and M. Edwards et al, "Measurement of the duration of x-ray lasing pumped by an optical laser pulse of picosecond duration," Optics Commun. 215, pp. 397-406, 2003.

[37] A. Klisnick, O. Guilbaud, and D. Ros et al, "Experimental study of the temporal coherence and spectral profile of the $13.9 \mathrm{~nm}$ transient x-ray laser," J. Quant. Spect. Rad. Trans. 99, pp. 370-380, 2006.

[38] L. M. Meng, D. Alessi, and O. Guilbaud et al, "Temporal coherence and spectral linewidth of an injectionseeded transient collisional soft x-ray laser," Optics Express 19, pp. 12087-12092, 2011.

[39] J. A. Koch, B. J. MacGowan, and L. B. DaSilva et al, "Observation of gain-narrowing and saturation behavior in Se x-ray laser line profiles," Phys. Rev. Lett. 68, pp. 3291-3294, 1992.

[40] P. X. Lu, E. Fill, and Y. L. Li et al, "Spatial coherence of prepulse-induced neonlike x-ray lasers," Phys. Rev. A 58, pp. 628-635, 1998.

[41] M. A. Larotonda, B. M. Luther, and Y. Wang et al, "Characteristic of a saturated $18.9 \mathrm{~nm}$ tabletop laser operating at 5-Hz repetition rate," IEEE J. Select. Topics Quant. Elect. 10, pp. 1363-1367, 2004.

[42] O. Guilbaud, A. Klisnick, and K. Cassou et al, "Origin of microstructures in picosecond x-ray laser beams," Europhys. Lett. 74, pp. 823-929, 2006.

[43] T. Ditmire, M. H. R. Hutchinson, and M. H. Key et al, "Amplification of XUV harmonic radiation in a Gallium amplifier," Phys. Rev. A 51, pp. R4337-R4340, 1995.

[44] C. L. S. Lewis, D. Neely, and D. M. O'Neill et al, "An injector amplifier double target configuration for the Ne-like Ge x-ray laser scheme," Optics Commun. 91, pp. 71-76, 1992.

[45] J. Zhang, J. Warwick, and E. Wolfrum et al, "Saturated output of a Ge XXIII x-ray laser at $19.6 \mathrm{~nm}$," Phys. Rev. A 54, pp. R4653-R4656, 1996. 